\title{
CO OBSERVATIONS OF THE 30-DORADUS REGION USING SEST
}

\author{
R.S. BOOTH \& L.E.B. JOHANSSON \\ Onsala Space Observatory \\ Chalmers University of Technology \\ S-43900 Onsala \\ Sweden
}

\begin{abstract}
Using the Swedish-ESO Submillimetre Telescope, we have mapped the CO (1-0) emission in two small areas of the LMC near 30 Dor. Some 20 molecular clouds have been identified and analysed. Cloud masses are calculated assuming virial equilibrium and the relationship between mass and $\mathrm{CO}$ luminosity is discussed.
\end{abstract}

\section{The observations}

The Magellanic Clouds are an important target for the Swedish-ESO Submillimetre telescope (SEST). Its $15 \mathrm{~m}$ aperture gives a beam-width of $44 \operatorname{arcsec}$ at $115 \mathrm{GHz}$, representing a linear resolution of $10 \mathrm{pc}$ at the distance of the LMC. Thus, for the first time we are able to observe individual molecular clouds in a nearby galaxy. This dramatic increase in resolution is demonstrated in Figure 1 which shows the contour map of Cohen et al. (1988) together with the maps of the regions observed by us (Johansson et al., 1989).

\section{Molecular cloud parameters}

We have isolated 23 clouds in these observations. Their half power diameters range in scale from about $5 \mathrm{pc}$ to $30 \mathrm{pc}$. The molecular masses of the clouds have been calculated, assuming virial equilibrium. This assumption may be justified from the approximate relationship between $\mathrm{CO}$ velocity dispersion $\sigma_{\mathrm{V}}$ and cloud size, $\sigma_{\mathrm{D}}, \sigma_{\mathrm{V}}=\sqrt{ } \sigma_{\mathrm{D}}$ (Johansson et al., 1989: Solomon et al., 1987). In this way we find that the molecular masses of the clouds range from 4 to $200 \cdot 10^{3} \mathrm{M}_{\mathrm{O}}$.

For giant molecular clouds in the Galaxy, Solomon et al. (1987) have found an empirical relation between virial mass and $\mathrm{CO}$ luminosity which they claim may be used to determine the mass of molecular gas in external galaxies from CO observations. Maloney and Black (1988) and Elmegreen (1989) have argued that metallicity and uv radiation flux may affect this conversion.

In view of this discussion, and concerning the low metallicity of the $\mathrm{LMC}$, it is interesting to plot the CO luminosity as a function of virial mass for the LMC clouds identified in this work. The result is shown in Figure 2 - the relationship found by Solomon et al. is plotted as a solid line on the figure. Figure 2 implies that the conversion between $\mathrm{L}_{\mathrm{CO}}$ and $\mathrm{M}_{\mathrm{H} 2}$ is different, on average by a factor of 2 at most, relative to the Galaxy. While at first sight, this result favours a constant conversion from galaxy to galaxy, Johansson et al., through observations of both the (1-0) and (2- 
1) rotational lines of ${ }^{12} \mathrm{CO}$ and ${ }^{13} \mathrm{CO}$, find that the ${ }^{12} \mathrm{CO}$ in the LMC has an optical depth $\tau \approx 1$. They therefore suggest that the $\mathrm{CO}$ clouds may indeed have suffered photodissociation and thus the volume occupied by detectable $\mathrm{CO}$ as a fraction of the total molecular volume (defined by the $\mathrm{H}_{2}$ ) may be smaller in this low metallicity system. Hence, the conversion factor from $\mathrm{CO}$ to $\mathrm{H}_{2}$ mass may be somewhat greater than 2 .

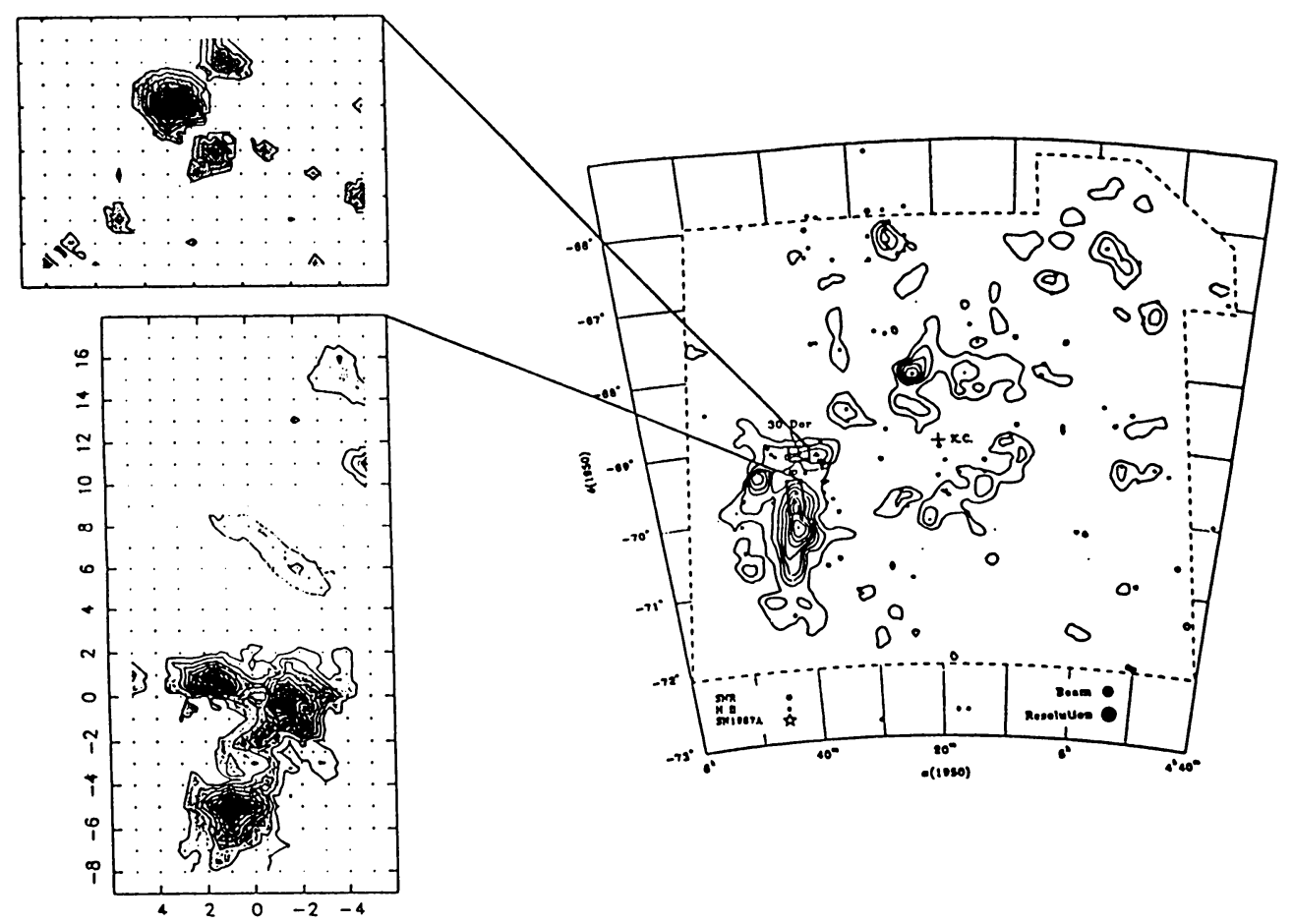

Figure 1. A map of the $\mathrm{CO}$ in the Large Magellanic Cloud (Cohen et al., 1988) showing the regions observed by Johannson et al. (1989) with the SEST telescope.

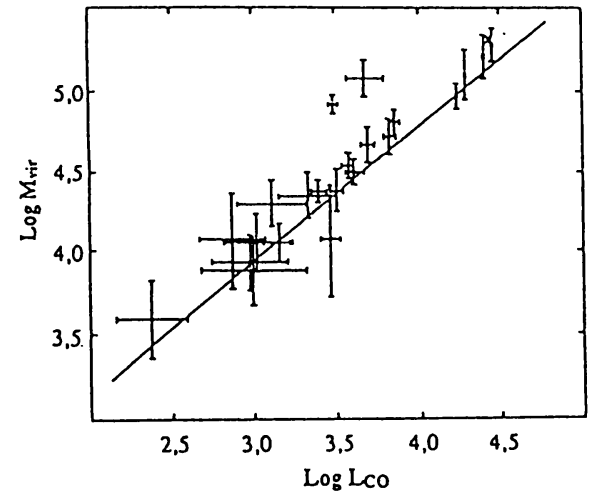

Figure 2. A plot of $\mathrm{L}_{\mathrm{CO}}$ v. virial mass for individual $\mathrm{CO}$ clouds in the LMC. The Galactic relation (Solomon et al., 1987) is shown as a solid line. 


\section{References}

Cohen, R.S., Dame, T.M., Garay, G., Montani, J., Rubio, M. and Thaddeus, P. (1988), Astrophys. J. 331, L95.

Elmegreen, B.G. (1989), Astrophys. J. 338, 178.

Johansson, L.E.B., Booth, R.S., Murphy, D., and Olberg, M. (1989), In Submillimetre Astronomy. Eds. G.D. Watt and A.S. Webster. Kluwer Academic Publishers.

Maloney, P. and Black, J.H. (1988), Astrophys. J. 325, 389.

Solomon, P.M., Rivolo, A., Barrett, J. and Yahil, A. (1987), Astrophys. J. 319, 730. 